\title{
Anterior communicating artery aneurysm presenting with vision loss
}

Dhaval P. Shukla, Dhananjaya I. Bhat, Bhagavatula I. Devi

Department of Neurosurgery, National Institute of Mental Health and Neurosciences, Bangalore, India

\section{ABSTRACT}

Anterior communicating artery aneurysm rarely presents with symptoms of compression of anterior visual pathways. We report a case of 65 years old man, who had complete loss of vision in right eye and temporal hemianopsia in left eye due to giant anterior communicating artery aneurysm.

Key words: Aneurysm, anterior communicating artery, optic nerve, vision loss

\section{Introduction}

The aneurysms originating from carotid-opthalmic artery, supraclinoid internal carotid artery, and internal carotid artery bifurcation are more likely to present with symptoms of anterior visual pathway compression. ${ }^{[1]} \mathrm{We}$ describe a rare case of anterior communicating aneurysm presenting with vision loss.

\section{Case Report}

A 65 years old, gentleman presented with headache of 3 months duration, and loss of vision in right eye of 15 days duration. He was suffering from non specific headache for which he did not seek medical attention. About 15 days back his vision decreased in right eye, which progressed to complete blindness within a week. He never had sudden onset of headache with vision loss. At admission he was conscious and alert. His best corrected visual acuity was no perception of light in right eye and 6/6 in left eye. He had relative afferent pupillary defect in right eye, and brisk pupillary reaction to light in left eye. The fundoscopy revealed

\begin{tabular}{|c|l|}
\hline \multicolumn{2}{|c|}{ Access this article online } \\
\hline Quick Response Code: & Website: \\
\hline & www.ruralneuropractice.com \\
\cline { 2 - 2 } & \\
\hline & DOI: \\
\hline
\end{tabular}

pale disc in right eye and normal disc in left eye. The visual fields assessment was done with computerized static visual field analysis using zeiss humphrey. The SITA FAST test was performed using goldmann parameters. Right eye could not be assessed because of poor vision. The left eye showed temporal hemianopsia. He did not have any other neurological deficits. The blood investigations revealed normal hematological and biochemical profile. The visual evoked potential revealed absence of all waves in right eye, and increased latency (N75-77.1 ms, P100-113.1 ms, and n145-157.8 $\mathrm{ms}$ ) in left eye. Unenhanced CT scan of head showed a hyperdense suprasellar lesion. The MRI of brain showed a well defined rounded suprasellar lesion measuring $26 \times 25 \times 23 \mathrm{~mm}$, which was composed of concentric rings of differing intensities, suggestive of partially thrombosed giant aneurysm. Post contrast MRI showed an aneurysm arising from anterior communicating artery (AComA), which was directed posteriorly [Figure 1]. Digital subtraction angiography (DSA) confirmed the findings on MRI [Figure 2]. The patient was offered surgery for securing aneurysm. As there was uncertainty about visual recovery he did not opt for any treatment and was discharged against medical advice.

\section{Discussion}

Large or giant aneurysm arising from anterior part of circle of Willis can result in visual symptoms as it is in close proximity to anterior visual pathways. The visual loss due to aneurysms is characterized by

Address for correspondence:

Dr. Dhaval Shukla, Department of Neurosurgery, National Institute of Mental Health and Neurosciences, Bangalore - 560 029 , India.

E-mail: neurodhaval@rediffmail.com 


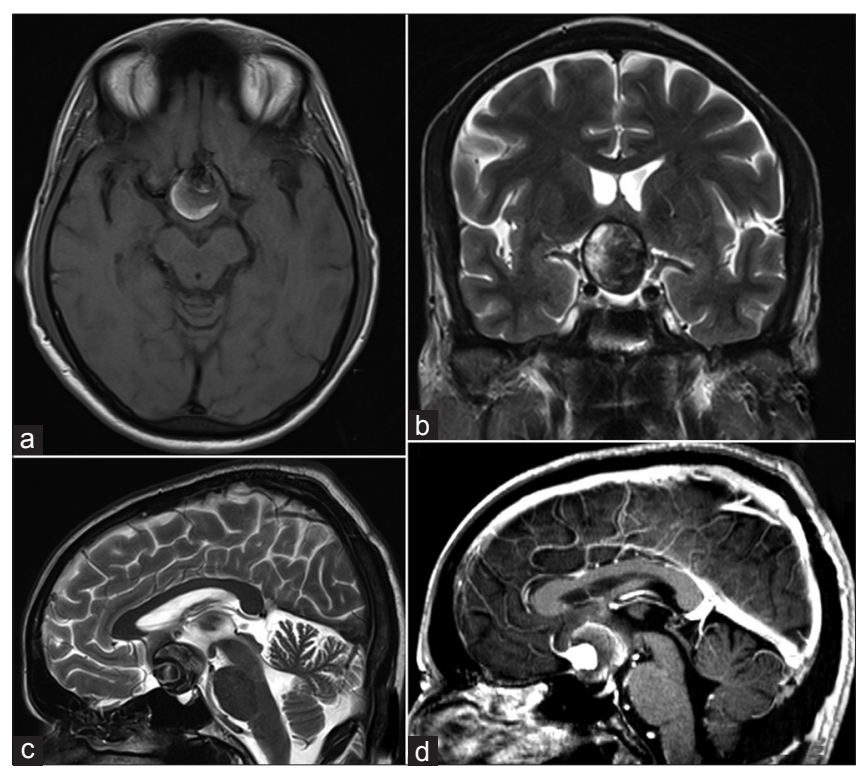

Figure 1: MRI of brain (a) T1W, axial section showing a rounded suprasellar lesion which is composed of alternate concentric rings of iso and hyperintensities (b) T2W, coronal section showing suprasellar lesion with hypointense rim and center of heterogeneous intensities (c) T2W, saggital section showing suprasellar lesion of mixed intensities (d) T1W, after contrast administration saggital section showing suprasellar lesion with brillian enhancement in center in continuity with anterior cerebral artery

fluctuation, unlike that from tumors where visual loss is gradually progressive. These fluctuations are due to thrombosis or intermittent dilatation of aneurysm. ${ }^{[1]}$ The common origins of aneurysms that present with symptoms of anterior visual pathway compression are carotid-opthalmic artery, supraclinoid internal carotid artery, and internal carotid artery bifurcation. Aneurysms arising from these arteries are often large or giant and can compress the visual apparatus. The AComA aneurysms rupture before becoming large enough to compress visual pathways, hence they present with subarachnoid hemorrhage rather than visual symptoms. ${ }^{[1]}$ Giant AComA aneurysms are extremely rare and may present with vision loss. ${ }^{[2]}$ Visual apparatus compression can occur from giant AComA aneurysm directed posteriorly and inferiorly as in our case. ${ }^{[1,2]}$ The pattern of visual loss in these cases is variable. The common pattern of visual loss is bilateral field deficits. ${ }^{[1]}$ Presentation with only monocular acute visual loss is very rare, and when it occurs, contralateral eye is more often affected. ${ }^{[3]}$ The other patterns of visual loss due to AComA aneurysms are central scotoma, bilateral heteronymous deficits, monocular or binocular inferior field loss, asymmetrical bitemporal hemianopsia, and incongruous homonymous hemianopsia. ${ }^{[1]}$ In our case, patient presented with subacute monocular visual loss. The opposite side temporal field defect was detected only on detailed visual field assessment, for which patient

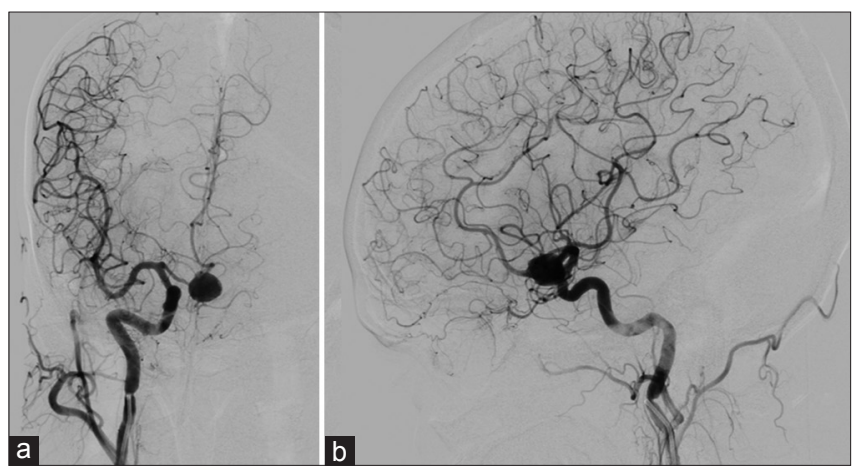

Figure 2: (a) DSA, right carotid injection, antero-posterior view, showing an aneurysm arising from anterior communicating artery directed inferiorly (b) DSA, lateral view, showing an aneurysm arising from anterior communicating artery directed posteriorly

was asymptomatic. The visual field deficit might have started with bitemporal hemianopsia due to chiasm compression, which would have not been noticed by the patient. The right optic nerve would have got compressed later, presenting with monocular visual loss. The common mechanism of visual loss is compression and ischemia due to giant aneurysm, as in our case, and direct hemorrhage in the optic nerve or chiasm due to rupture of smaller aneurysm..$^{[1]}$ The penetration, splitting, or fenestration of optic nerves by aneurysms is extremely rare..$^{[4]}$

The predictability of recovery of vision is poor in case of aneurysmal visual loss. In a review of 165 cases of aneurysmal visual loss, visual function improved in $70 \%$ cases, remained unchanged in $21 \%$, and worsened in $9 \%$ after surgical clipping. There was no significant relationship between visual recovery and aneurysm location, aneurysm size, or presence of subarachnoid hemorrhage. Surgical clipping was significantly associated with better visual outcome as compare to endovascular coiling. ${ }^{[5]}$ As the numbers of cases of visual loss due to AComA are rare the exact figure of visual outcome is not available. ${ }^{[6]}$ The results of treatment are variable ranging from no improvement to complete recovery. ${ }^{[7]} \mathrm{A}$ patient with only a direct compression of the nerve by the aneurysm with no bleed can be expected to improve after surgery. ${ }^{[3]}$

\section{References}

1. Kasner SE, Liu GT, Galetta S. Neuroophthalmologic aspects of aneurysms. Neuroimaging Clin N Am 1997;7:679-92.

2. Lownie SP, Drake CG, Peerless SJ, Ferguson GG, Pelz DM. Clinical presentation and management of giant anterior communicating artery region aneurysms. J Neurosurg 2000;92:267-77.

3. Bhat DI, Sampath S. Anterior communicating artery aneurysm presenting as monocular blindness. Br J Neurosurg 2011;25:644-6.

4. Horiuchi T, Uchiyama T, Kusano Y, Okada M, Hongo K, Kobayashi S. Penetration of the optic nerve or chiasm by anterior communicating artery 
aneurysms: Three case reports. Neuro-ophthalmology 2011;35:128-32.

5. Schuss P, Güreseir E, Berkefeld J, Seifert V, Vatter H. Influence of surgical or endovascular treatment on visual symptoms caused by intracranial aneurysms: Single-center series and systematic review. J Neurosurg 2011;115:694-9.

6. de Oliveira JG, Borba LA, Rassi-Neto A, de Moura SM, Sanchez-Júnior SL, Rassi MS, et al. Intracranial aneurysms presenting with mass effect over the anterior optic pathways: Neurosurgical management and outcomes. Neurosurg Focus 2009;26:E3.
7. Chan JW, Hoyt WF, Ellis WG, Gress D. Pathogenesis of acute monocular blindness from leaking anterior communicating artery aneurysms: Report of six cases. Neurology 1997;48:680-3.

How to cite this article: Shukla DP, Bhat DI, Devi BI. Anterior communicating artery aneurysm presenting with vision loss. J Neurosci Rural Pract 2013;4:305-7.

Source of Support: Nil. Conflict of Interest: None declared. 